CUBO A Mathematical Journal

Vol.12, № 03, (35-48). October 2010

\title{
Measure of Noncompactness and Nondensely Defined Semilinear Functional Differential Equations with Fractional Order
}

\author{
MOUFFAK BENCHOHRA ${ }^{1}$ \\ Laboratoire de Mathématiques, Université de Sidi Bel-Abbès, \\ B.P. 89, 22000, Sidi Bel-Abbès, Algérie \\ email: benchohra@univ-sba.dz \\ GASTON M. N'GUÉRÉKATA \\ Department of Mathematics, Morgan State University, \\ 1700 E. Cold Spring Lane, Baltimore M.D. 21252, USA \\ email: Gaston. N'Guerekata@morgan.edu \\ AND \\ DJAMILA SEBA \\ Département de Mathématiques, Université de Boumerdès, \\ Avenue de l'indépendance, 35000 Boumerdès, Algérie \\ email: djam_seba@yahoo.fr
}

\begin{abstract}
This paper is devoted to study the existence of integral solutions for a nondensely defined semilinear functional differential equations involving the Riemann-Liouville derivative in
\end{abstract}

\footnotetext{
${ }^{1}$ Corresponding author
} 
a Banach space. The arguments are based upon Mönch's fixed point theorem and the technique of measures of noncompactness.

\title{
RESUMEN
}

\begin{abstract}
Este artículo es dedicado al estudio de existencia de soluciones integrales para ecuaciones diferenciales funcionales semilineales envolviendo la derivada de Riemann-Liouville en un espacio de Banach. Los argumentos se basan en un teorema de punto fijo de Mönch y la técnica de no compacidad.
\end{abstract}

Key words and phrases: Partial differential equations, fractional derivative, fractional integral, fixed point, semigroups, integral solutions, finite delay, measure of noncompactness, fixed point, Banach space.

Math. Subj. Class.: 34G20, 34G25, 26A33, $26 A 42$.

\section{Introduction}

The theory of functional differential equations has emerged as an important branch of nonlinear analysis. It is worthwhile mentioning that several important problems of the theory of ordinary and delay differential equations lead to investigations of functional differential equations of various types, see the books of Hale and Verduyn Lunel [22], Kolmanovskii and Myshkis [26], Wu [42], and the references therein. On the other hand the theory of fractional differential equations is also intensively studied and finds numerous applications in describing real world problems (see for instance the monographs of Lakshmikantham et al. [27], Kilbas et al. [25], Miller and Ross [31], Podlubny [39], Samko et al. [40], and the papers of Agarwal et al. [1], Benchohra et al. [11, 12], Chang and Nieto [14], Diethelm et al. [16], Furati and Tatar [17, 18], Gaul et al. [19], Glockle and Nonnenmacher [20], Lakshmikantham and Devi [28], Mainardi [29], Metzler et al. [30], N'Guérékata et al [33, 34, 35], and the references therein). Jaradat et al. [23], studied the existence and uniqueness of mild solutions for a class of initial value problem for a semilinear integrodifferential equation involving the Caputo's fractional derivative.

In this paper we will examine the following semilinear functional differential equation of fractional order

$$
\begin{gathered}
D^{r} y(t)=A y(t)+f\left(t, y_{t}\right), \quad t \in J=[0, b], \quad r>0 \\
y(t)=\phi(t), \quad t \in[-\rho, 0],
\end{gathered}
$$

where $D^{r}$ is the standard Riemann-Liouville fractional derivative, $f: J \times C([-\rho, 0], E) \rightarrow E$ is a given function, $A: D(A) \subset E \rightarrow E$ is a nondensely defined closed linear operator on $E$. 
$\phi:[-\rho, 0] \rightarrow E$ a given continuous function with $\phi(0)=0$ and $(E,|\cdot|)$ a Banach space. For any function $y$ defined on $[-\rho, b]$ and any $t \in J$ we denote by $y_{t}$ the element of $C([-\rho, 0], E)$ defined by

$$
y_{t}(\theta)=y(t+\theta), \theta \in[-\rho, 0] .
$$

Here $y_{t}(\cdot)$ represents the history of the state from time $t-\rho$, up to the present time $t$.

Let us mention that the functional differential equation of the type (1) was investigated, in the case when $A$ generates a $C_{0}$-semigroup, in a lot of papers and developed with the help of various tools of fixed-point theory see, for instance Belmekki et al. [8, 9, 10].

The principal goal of this paper is to extend such results to the case when the operator $A$ is nondensely defined and satisfies the Hille-Yosida condition, and to initiate the application of the technique of measures of noncompactness to investigate the problem of the existence of integral solutions for (1)-(2). Especially that technique combined with an appropriate fixed point theorem has proved to be a very useful tool in the study of the existence of solutions for several types of integral and differential equations; see for example Alvàrez [3], Banas̀ et al. [5, 6, 7], Benchohra et al. [13], Guo et al. [21], Mönch [32], Mönch and Von Harten [37], and Szufla [41].

\section{Preliminaries}

In this section we collect some definitions, notations and results needed in the sequel. At first, we recall the definition of Riemann-Liouville fractional primitive and fractional derivative.

Denote by $C(J, E)$ the Banach space of continuous functions $J \rightarrow E$, with the usual supremum norm

$$
\|y\|_{\infty}=\sup \{|y(t)|, \quad t \in J\} .
$$

For $\psi \in C([-\rho, 0], E)$ the norm of $\psi$ is defined by

$$
\|\psi\|_{\mathscr{C}}=\sup \{|\psi(\theta)|, \theta \in[-\rho, 0]\} .
$$

$B(E)$ denotes the Banach space of all bounded linear operators from $E$ into $E$, with norm

$$
\|N\|_{B(E)}=\sup \{|N(y)|:|y|=1\} .
$$

Let $L^{1}(J, E)$ be the Banach space of measurable functions $y: J \rightarrow E$ which are Bochner integrable, equipped with the norm

$$
\|y\|_{L^{1}}=\int_{J}|y(t)| d t
$$

Let $L^{\infty}(J, E)$ be the Banach space of measurable functions $y: J \rightarrow E$ which are bounded, equipped with the norm

$$
\|y\|_{L^{\infty}}=\inf \{c>0:\|y(t)\| \leq c, \text { a.e. } t \in J\} .
$$


For a given set $V$ of functions $v:[-\rho, b] \rightarrow E$, let us denote by

$$
V(t)=\{v(t): v \in V\}, t \in[-\rho, b]
$$

and

$$
V(J)=\{v(t): v \in V, t \in[-\rho, b]\} .
$$

Definition 2.1. ([25, 39]). The Riemann-Liouville fractional primitive of order $r>0$ of a function $h:(0, b] \rightarrow E$ is defined by

$$
I_{0}^{r} h(t)=\frac{1}{\Gamma(r)} \int_{0}^{t}(t-s)^{r-1} h(s) d s,
$$

provided the right side is pointwise defined on $(0, b]$, and where $\Gamma$ is the gamma function.

Definition 2.2. ([25, 39]). The Riemann-Liouville fractional derivative of order $r \in(0,1]$ of a continuous function $h:(0, b] \rightarrow E$ is defined by

$$
\begin{aligned}
\frac{d^{r} h(t)}{d t^{r}} & =\frac{1}{\Gamma(1-r)} \frac{d}{d t} \int_{0}^{t}(t-s)^{-r} h(s) d s \\
& =\frac{d}{d t} I_{0}^{1-r} h(t) .
\end{aligned}
$$

Definition 2.3. A map $f: J \times C([-\rho, 0], E) \rightarrow E$ is said to be Carathéodory if

(i) $t \longmapsto f(t, u)$ is measurable for each $u \in C([-\rho, 0], E)$;

(ii) $u \longmapsto F(t, u)$ is continuous for almost each $t \in J$.

For completeness we gather some definitions and basic facts of integrated semigroups, and operators satisfying Hille-Yosida condition.

Definition 2.4. [4]. Let $E$ be a Banach space. An integrated semigroup is a family of operators $(S(t))_{t \geq 0}$ of bounded linear operators $S(t)$ on $E$ with the following properties:

(i) $S(0)=0$;

(ii) $t \rightarrow S(t)$ is strongly continuous;

(iii) $S(s) S(t)=\int_{0}^{s}(S(t+\tau)-S(\tau)) d \tau$, for all $t, s \geq 0$.

Definition 2.5. [24]. An operator A is called a generator of an integrated semigroup if there exists $\omega \in \mathbb{R}$ such that $(\omega, \infty) \subset \rho_{0}(A)\left(\rho_{0}(A)\right.$, is the resolvent set of $\left.A\right)$ and there exists a strongly continuous exponentially bounded family $(S(t))_{t \geq 0}$ of bounded operators such that $S(0)=0$ and $R(\lambda, A):=(\lambda I-A)^{-1}=\lambda \int_{0}^{\infty} e^{-\lambda t} S(t) d t$ exists for all $\lambda$ with $\lambda>\omega$. 
Proposition 2.1. [4]. Let $A$ be the generator of an integrated semigroup $(S(t))_{t \geq 0}$. Then for all $x \in E$ and $t \geq 0$,

$$
\int_{0}^{t} S(s) x d s \in D(A) \text { and } S(t) x=A \int_{0}^{t} S(s) x d s+t x .
$$

Definition 2.6. We say that the linear operator A satisfies the Hille-Yosida condition if there exists $M \geq 0$ and $\omega \in \mathbb{R}$ such that $(\omega, \infty) \subset \rho_{0}(A)$ and

$$
\sup \left\{(\lambda-\omega)^{n}\left|(\lambda I-A)^{-n}\right|: n \in \mathbb{N}, \lambda>\omega\right\} \leq M .
$$

Definition 2.7. [24].

(i) An integrated semigroup $(S(t))_{t \geq 0}$ is called locally Lipschitz continuous if, for all $\tau>0$, there exists a constant $L$ such that

$$
|S(t)-S(s)| \leq L|t-s|, \quad t, s \in[0, \tau] .
$$

(ii) An integrated semigroup $(S(t))_{t \geq 0}$ is called non degenerate if $S(t) x=0$, for all $t \geq 0$, implies that $x=0$.

Theorem 2.1. [24]. The following assertions are equivalent:

(i) A is the generator of a non degenerate, locally Lipschitz continuous integrated semigroup;

(ii) A satisfies the Hille-Yosida condition.

If $A$ is the generator of an integrated semigroup $(S(t))_{t \geq 0}$ which is locally Lipschitz, then from [4], $S(\cdot) x$ is continuously differentiable if and only if $x \in \overline{D(A)}$ and $\left(S^{\prime}(t)\right)_{t \geq 0}$ is a $C_{0}$-semigroup on $\overline{D(A)}$.

Let $(S(t))_{t \geq 0}$ be the integrated semigroup generated by $A$. We note that, if $A$ satisfies the Hille-Yosida condition, then $\left\|S^{\prime}(t)\right\|_{B(E)} \leq M e^{\omega t}, t \geq 0$, where $M$ and $\omega$ are the constants considered in the Hille-Yosida condition.

Now let us recall some fundamental facts of the notion of Kuratowski measure of noncompactness.

Definition 2.8. ([6]) Let $E$ be a Banach space and $\Omega_{E}$ the bounded subsets of $E$. The Kuratowski measure of noncompactness is the map $\alpha: \Omega_{E} \rightarrow[0, \infty]$ defined by

$$
\alpha(B)=\inf \left\{\epsilon>0: B \subseteq \cup_{i=1}^{n} B_{i} \text { and } \operatorname{diam}\left(B_{i}\right) \leq \epsilon\right\} \text {; here } B \in \Omega_{E} .
$$


Properties: The Kuratowski measure of noncompactness satisfies the following properties (for more details see [6]).

(a) $\alpha(B)=0 \Leftrightarrow \bar{B}$ is compact ( $B$ is relatively compact).

(b) $\alpha(B)=\alpha(\bar{B})$.

(c) $A \subset B \Rightarrow \alpha(A) \leq \alpha(B)$.

(d) $\alpha(A+B) \leq \alpha(A)+\alpha(B)$

(e) $\alpha(c B)=|c| \alpha(B) ; c \in \mathbb{R}$.

(f) $\alpha(\operatorname{conv} B)=\alpha(B)$.

Theorem 2.2. ([2, 32]) Let $D$ be a bounded, closed and convex subset of a Banach space such that $0 \in D$, and let $N$ be a continuous mapping of $D$ into itself. If the implication

$$
V=\overline{\operatorname{conv}} N(V) \quad \text { or } \quad V=N(V) \cup\{0\} \Rightarrow \alpha(V)=0
$$

holds for every subset $V$ of $D$, then $N$ has a fixed point.

Lemma 2.1. ([41]) Let $D$ be a bounded, closed and convex subset of the Banach space $C(J, E)$, $G$ a continuous function on $J \times J$ and $f$ a function from $J \times C([-\rho, 0], E) \rightarrow E$ which satisfies the Carathéodory conditions and there exists $p \in L^{1}\left(J, \mathbb{R}_{+}\right)$such that for each $t \in J$ and each bounded set $B \subset C([-\rho, 0], E)$ we have

$$
\lim _{k \rightarrow 0^{+}} \alpha\left(f\left(J_{t, k} \times B\right)\right) \leq p(t) \alpha(B) ; \text { here } J_{t, k}=[t-k, t] \cap J .
$$

If $V$ is an equicontinuous subset of $D$, then

$$
\alpha\left(\left\{\int_{J} G(s, t) f\left(s, y_{s}\right) d s: y \in V\right\}\right) \leq \int_{J}\|G(t, s)\| p(s) \alpha(V(s)) d s .
$$

\section{Main Results}

We start with the following principal assumption and the definition of integral solutions to the problem (1)-(2).

(H1) A satisfies the Hille-Yosida condition.

Definition 3.1. We say that a continuous function $y:[-\rho, b] \rightarrow E$ is an integral solution of problem (1)-(2) if 
(i) $\int_{0}^{t}(t-s)^{r-1} y(s) d s \in D(A)$ for $t \in J$,

(ii) $y(t)=\phi(t), t \in[-\rho, 0]$, and

(iii) $y(t)=\frac{1}{\Gamma(r)} A \int_{0}^{t}(t-s)^{r-1} y(s) d s+\frac{1}{\Gamma(r)} \int_{0}^{t}(t-s)^{r-1} f\left(s, y_{s}\right) d s, t \in J$.

From the definition it follows that $y(t) \in \overline{D(A)}, \forall t \geq 0$. Moreover, $y$ satisfies the following variation of constants formula:

$$
y(t)=\frac{1}{\Gamma(r)} \frac{d}{d t} \int_{0}^{t} S(t-s)(t-s)^{r-1} f\left(s, y_{s}\right) d s, \quad t \geq 0 .
$$

Let $B_{\lambda}=\lambda R(\lambda, A)$, then for all $x \in \overline{D(A)}, B_{\lambda} x \mapsto x$ as $\lambda \mapsto \infty$.

We notice also that, if $y$ satisfies (3), then

$$
y(t)=\lim _{\lambda \rightarrow \infty} \frac{1}{\Gamma(r)} \int_{0}^{t} S^{\prime}(t-s)(t-s)^{r-1} B_{\lambda} f\left(s, y_{s}\right) d s, \quad t \geq 0 .
$$

Without lost of generality, we will assume that $w>0$.

Let us list some conditions on the functions involved in the problem (1)-(2).

(H2) The operator $S^{\prime}(t)$ is compact in $\overline{D(A)}$ whenever $t>0$ and

$$
\left\|S^{\prime}(t)\right\|_{B(E)} \leq M e^{\omega t}, t \in J .
$$

(H3) $f: J \times C([-\rho, 0], E) \rightarrow E$ is of Carathéodory.

(H4) There exists a function $p \in L^{\infty}\left(J, \mathbb{R}_{+}\right)$such that

$$
|f(t, u)| \leq p(t)\left(\|u\|_{C}+1\right) \text {, for a.e. } t \in J, \text { and each } u \in C([-\rho, 0], E) .
$$

(H5) For almost each $t \in J$ and each bounded set $B \subset C([-\rho, 0], E)$ we have

$$
\lim _{h \rightarrow 0^{+}} \alpha\left(f\left(J_{t, h} \times B\right)\right) \leq p(t) \alpha(B) ; \text { here } J_{t, h}=[t-h, t] \cap J .
$$

(H6) Assume

$$
\frac{M b^{r} p^{*} e^{\omega b}}{\Gamma(r+1)}<1
$$

Let $p^{*}=\|p\|_{L^{\infty}}$. Our main result reads as follows

Theorem 3.1. Assume that assumptions (H1)-(H6) hold. Then the the problem (1)-(2) has at least one integral solution. 
Proof. We shall reduce the existence of solutions of (1)-(2) to a fixed point problem. Consider the operator $N: C([-\rho, b], E) \rightarrow C([-\rho, b], E)$ defined by

$$
N(y)(t)= \begin{cases}\phi(t), & t \in[-\rho, 0] \\ \frac{1}{\Gamma(r)} \frac{d}{d t} \int_{0}^{t}(t-s)^{r-1} S(t-s) f\left(s, y_{s}\right) d s, & t \in[0, b] .\end{cases}
$$

Let $r_{0}>0$ be such that

$$
r_{0} \geq \frac{M p^{*} b^{r} e^{\omega b}}{\Gamma(r+1)-M b^{r} p^{*} e^{\omega b}}
$$

and consider the set

$$
D_{r_{0}}=\left\{y \in C([-\rho, b], E):\|y\|_{\infty} \leq r_{0}\right\} .
$$

Clearly, the subset $D_{r_{0}}$ is closed, bounded and convex. We shall show that $N$ satisfies the assumptions of Theorem 2.2. The proof will be given in three steps.

Step 1: $N$ is continuous.

Let us consider a sequence $\left\{y_{n}\right\}$ such that $y_{n} \rightarrow y$ in $C([-\rho, b], E)$. Then for each $t \in J$

$$
\begin{aligned}
\left|N\left(y_{n}\right)(t)-N(y)(t)\right| & =\left|\frac{1}{\Gamma(r)} \frac{d}{d t} \int_{0}^{t}(t-s)^{r-1} S(t-s)\left[f\left(s, y_{n_{s}}\right)-f\left(s, y_{s}\right)\right] d s\right| \\
& \leq \frac{M e^{\omega t}}{\Gamma(r)} \int_{0}^{t} e^{-\omega s}(t-s)^{r-1}\left|f\left(s, y_{n_{s}}\right)-f\left(s, y_{s}\right)\right| d s \\
& \leq \frac{M e^{\omega b}}{\Gamma(r)} \int_{0}^{t}(t-s)^{r-1}\left|f\left(s, y_{n_{s}}\right)-f\left(s, y_{s}\right)\right| d s .
\end{aligned}
$$

Let $\mu>0$ be such that

$$
\left\|y_{n}\right\|_{\infty} \leq \mu,\|y\|_{\infty} \leq \mu .
$$

By (H4) we have

$$
\left|(t-s)^{r-1}\left[f\left(s, y_{n_{s}}\right)-f\left(s, y_{s}\right)\right]\right| \leq 2 p^{*}(\mu+1)(t-s)^{r-1} \in L^{1}\left(J, \mathbb{R}_{+}\right) .
$$

Since $f$ is a Carathéodory function, the Lebesgue dominated convergence theorem implies that

$$
\left\|N\left(y_{n}\right)-N(y)\right\|_{\infty} \rightarrow 0 \quad \text { as } n \rightarrow \infty
$$

Step 2: $N$ maps $D_{r_{0}}$ into itself.

For each $y \in D_{r_{0}}$, by (H4) and (H6) we have for each $t \in J$

$$
|N(y)(t)|=\left|\frac{1}{\Gamma(r)} \frac{d}{d t} \int_{0}^{t}(t-s)^{r-1} S(t-s) f\left(s, y_{s}\right) d s\right|
$$




$$
\begin{aligned}
& \leq \frac{M b^{r} p^{*} e^{\omega b}\left(r_{0}+1\right)}{\Gamma(r+1)} \\
& \leq r_{0}
\end{aligned}
$$

Step 3: $N\left(D_{r_{0}}\right)$ is bounded and equicontinuous.

By Step 2, it is obvious that $N\left(D_{r_{0}}\right) \subset D_{r_{0}}$ is bounded.

For the equicontinuity of $N\left(D_{r_{0}}\right)$. Let $\tau_{1}, \tau_{2} \in J, \tau_{1}<\tau_{2}$, thus if $\epsilon>0$ and $\epsilon \leq \tau_{1} \leq \tau_{2}$ we have for any $y \in D_{r_{0}}$;

$$
\begin{aligned}
\left|N(y)\left(\tau_{2}\right)-N(y)\left(\tau_{1}\right)\right|= & \mid \lim _{\lambda \rightarrow \infty} \frac{1}{\Gamma(r)} \int_{0}^{\tau_{2}}\left(\tau_{2}-s\right)^{r-1} S^{\prime}\left(\tau_{2}-s\right) B_{\lambda} f\left(s, y_{s}\right) d s \\
- & \lim _{\lambda \rightarrow \infty} \frac{1}{\Gamma(r)} \int_{0}^{\tau_{1}}\left(\tau_{1}-s\right)^{r-1} S^{\prime}\left(\tau_{1}-s\right) B_{\lambda} f\left(s, y_{s}\right) d s \mid \\
\leq & M p^{*}\left(r_{0}+1\right)\left(\frac{1}{\Gamma(r)} \int_{0}^{\tau_{1}-\epsilon}\left[\left(\tau_{2}-s\right)^{r-1}-\left(\tau_{1}-s\right)^{r-1}\right] d s\right. \\
+ & \left\|S^{\prime}\left(\tau_{2}-\tau_{1}+\epsilon\right)-S^{\prime}(\epsilon)\right\|_{B(E)}\left\{\frac{1}{\Gamma(r)} \int_{0}^{\tau_{1}-\epsilon}\left(\tau_{2}-s\right)^{r-1} d s\right\} \\
+ & \frac{1}{\Gamma(r)} \int_{\tau_{1}-\epsilon}^{\tau_{1}}\left[\left(\tau_{2}-s\right)^{r-1}-\left(\tau_{1}-s\right)^{r-1}\right] d s \\
+ & \left\|S^{\prime}\left(\tau_{2}-\tau_{1}\right)-I\right\|_{B(E)}\left\{\frac{1}{\Gamma(r)} \int_{\tau_{1}-\epsilon}^{\tau_{1}}\left(\tau_{2}-s\right)^{r-1} d s\right\} \\
& \left.+\frac{1}{\Gamma(r)} \int_{\tau_{1}}^{\tau_{2}}\left(\tau_{2}-s\right)^{r-1} d s\right) .
\end{aligned}
$$

As $\tau_{1} \rightarrow \tau_{2}$ and $\epsilon$ sufficiently small, the right-hand side of the above inequality tends to zero, since $S^{\prime}(t)$ is a strongly continuous operator and the compactness of $S^{\prime}(t)$ for $t>0$ implies the continuity in the uniform operator topology (see [38]).

Now let $V$ be a subset of $D_{r_{0}}$ such that $V \subset \overline{c o n v}(N(V) \cup\{0\})$.

$V$ is bounded and equicontinuous and therefore the function $v \rightarrow v(t)=\alpha(V(t))$ is continuous on $[-\rho, b]$. By (H5), Lemmas 2.1 and the properties of the measure $\alpha$ we have for each $t \in$ $[-\rho, b]$

$$
\begin{aligned}
v(t) & \leq \alpha(N(V)(t) \cup\{0\}) \\
& \leq \alpha(N(V)(t)) \\
& \leq \lim _{\lambda \rightarrow \infty} \frac{1}{\Gamma(r)} \int_{0}^{t}(t-s)^{r-1} S^{\prime}(t-s) B_{\lambda} p(s) \alpha(V(s)) d s
\end{aligned}
$$




$$
\begin{aligned}
& \leq \frac{M p^{*} e^{\omega t}}{\Gamma(r)} \int_{0}^{t}(t-s)^{r-1} v(s) d s \\
& \leq\|v\|_{\infty} \frac{M b^{r} p^{*} e^{\omega b}}{\Gamma(r+1)} .
\end{aligned}
$$

This means that

$$
\|v\|_{\infty}\left(1-\frac{M b^{r} p^{*} e^{\omega b}}{\Gamma(r+1)}\right) \leq 0 .
$$

By (H6) it follows that $\|v\|_{\infty}=0$, that is $v(t)=0$ for each $t \in[-\rho, b]$, and then $V(t)$ is relatively compact in $E$. In view of the Ascoli-Arzelà theorem, $V$ is relatively compact in $D_{r_{0}}$. Applying now Theorem 2.2 we conclude that $N$ has a fixed point which is an integral solution for the problem (1)-(2).

\section{An Example}

As an application of our results we consider the following fractional time partial functional differential equation of the form

$$
\begin{gathered}
\frac{\partial^{\alpha}}{\partial t^{\alpha}} z(t, x)=\frac{\partial^{2}}{\partial x^{2}} z(t, x)+Q(t, z(t-r, x)), x \in[0, \pi], t \in[0,1], \alpha \in(0,1], \\
z(t, 0)=z(t, \pi)=0, t \in[0,1] \\
z(t, x)=\varphi(t, x), t \in[-r, 0], x \in[0, \pi]
\end{gathered}
$$

where $r>0, \varphi:[-r, 0] \times[0, \pi] \rightarrow \mathbb{R}$ is continuous and $Q:[0,1] \times \mathbb{R} \rightarrow \mathbb{R}$ is a given function.

Let

$$
\begin{gathered}
y(t)(x)=z(t, x), t \in J, x \in[0, \pi], \\
\phi(\theta)(x)=\varphi(\theta, x), \theta \in[-r, 0], x \in[0, \pi], \\
F(t, \phi)(x)=Q(t, \varphi(\theta, x)), \theta \in[-r, 0], x \in[0, \pi] .
\end{gathered}
$$

We choose $E=C([0, \pi] ; \mathbb{R})$ endowed with the uniform topology and consider the operator $A: D(A) \subset E \rightarrow E$ defined by:

$$
D(A)=\left\{y \in C^{2}([0, \pi], \mathbb{R}): y(0)=y(\pi)=0\right\} \quad A y=y^{\prime \prime} .
$$

It is well known (see [15]) that the operator $A$ Satisfies the Hille-Yosida condition with $(0,+\infty) \subset \rho(A),\left\|(\lambda I-A)^{-1}\right\| \leq \frac{1}{\lambda}$ for $\lambda>0$, and

$$
\overline{D(A)}=\{y \in E ; y(0)=y(\pi)=0\} \neq E .
$$


It follows that $A$ generates an integrated semigroup $(S(t))_{t \geq 0}$ and $\left\|S^{\prime}(t)\right\| \leq e^{-\mu t}$ for $t \geq 0$ and for some constant $\mu>0$. We can show that problem (1)-(2) is an abstract formulation of problem (4)-(6).

Assume that the function $Q$ satisfies the following conditions

(i) The function $Q: J \times \mathbb{R} \rightarrow \mathbb{R}$ is of Carathéodory.

(ii) $|Q(t, z)| \leq \frac{1}{e^{t+2}}(|z|+1)$ for each $(t, z) \in J \times \mathbb{R}$.

It is clear that conditions (H1)-(H4) are satisfied. We shall show that (H6) holds with

$$
\begin{gathered}
p(t)=\frac{1}{e^{t+2}}, t \in[0,1], \\
M=1, b=1, p^{*}=\frac{1}{e^{2}} .
\end{gathered}
$$

Indeed, we have

$$
\frac{M b^{r} p^{*} e^{\omega b}}{\Gamma(r+1)} \leq \frac{1}{e^{2} \Gamma(r+1)}<1 \text {, for each } r \in(0,1] .
$$

Hence, Theorem 3.1 implies that problem (4)-(6) has an integral solution $z$ on $[-r, 1] \times$ $[0, \pi]$.

\section{References}

[1] Agarwal, R.P., Benchohra, M. And Hamani, S., Boundary value problems for differential inclusions with fractional order, Adv. Stud. Contemp. Math., 12 (2) (2008), 181196.

[2] Agarwal, R.P., Meehan, M. And O'Regan, D., Fixed Point Theory and Applications, Cambridge University Press, Cambridge, 2001.

[3] ALVÀreZ, J.C., Measure of noncompactness and fixed points of nonexpansive condensing mappings in locally convex spaces, Rev. Real. Acad. Cienc. Exact. Fis. Natur. Madrid, 79 (1985), 53-66.

[4] ARendt, W., Vector valued Laplace transforms and Cauchy problems, Israel J. Math., 59 (1987), 327-352.

[5] BANAS̀, K. AND DhaGe, B.C., Global asymptotic stability of solutions of a fractional integral equation, Nonlinear Anal., 69 (2008) 1945-1952. 
[6] Banas̀, J. AND Goebel, K., Measures of Noncompactness in Banach Spaces, Marcel Dekker, New York, 1980.

[7] BANAS̀, J. AND SADARANGANI, K., On some measures of noncompactness in the space of continuous functions, Nonlinear Anal., 68 (2008), 377-383.

[8] BelmekKi, M. AND Benchohra, M., Existence results for fractional order semilinear functional differential equations, Proc. A. Razmadze Math. Inst., 146 (2008), 9-20.

[9] Belmekri, M., Benchohra, M. And Gorniewicz, L., Semilinear functional differential equations with fractional order and infinite delay, Fixed Point Theory, 9 (2) (2008), 423-439.

[10] Belmekki, M., Benchohra, M., Gòrniewicz, L. And Ntouyas, S.K., Existence results for semilinear perturbed functional differential inclusions with infinite delay, Nonlinear Anal. Forum, 13 (2) (2008), 135-165.

[11] Benchohra, M., Graef, J.R. And Hamani, S., Existence results for boundary value problems with nonlinear fractional differential equations, Appl. Anal., 87 (7) (2008), 851-863.

[12] Benchohra, M., Hamani, S. And Ntouyas, S.K., Boundary value problems for differential equations with fractional order, Surv. Math. Appl., 3 (2008), 1-12.

[13] Benchohra, M., Henderson, J. And Seba, D., Measure of noncompactness and fractional differential equations in Banach spaces, Commun. Appl. Anal., 12 (4) (2008), 419428.

[14] Chang, Y.-K. AND Nieto, J.J., Some new existence results for fractional differential inclusions with boundary conditions, Math. Comput. Model., 49 (2009), 605-609.

[15] Da Prato, G. AND Sinestrari, E., Differential operators with non-dense domains, Ann. Scuola. Norm. Sup. Pisa Sci., 14 (1987), 285-344.

[16] Diethelm, K. AND Ford, N.J., Analysis of fractional differential equations, J. Math. Anal. Appl., 265 (2002), 229-248.

[17] Furati, K.M. AND TAtaR, N.-EDdine, Behavior of solutions for a weighted Cauchytype fractional differential problem, J. Fract. Calc., 28 (2005), 23-42.

[18] Furati, K.M. AND TATAR, N.-EDdine, Power type estimates for a nonlinear fractional differential equation, Nonlinear Anal., 62 (2005), 1025-1036.

[19] Gaul, L., Klein, P. And Kempfle, S., Damping description involving fractional operators, Mech. Systems Signal Processing, 5 (1991), 81-88. 
[20] Glockle, W.G. And Nonnenmacher, T.F., A fractional calculus approach of selfsimilar protein dynamics, Biophys. J., 68 (1995), 46-53.

[21] Guo, D., Lakshmikantham, V. And LiU, X., Nonlinear Integral Equations in Abstract Spaces, Kluwer Academic Publishers Group, Dordrecht, 1996.

[22] Hale, J.K. ANd Lunel, S.V., Introduction to Functional -Differential Equations, Springer-Verlag, New York, 1993.

[23] Jaradat, O.K., Al-Omari, A. And Momani, S., Existence of mild solution for fractional semilinear initial value problems, Nonlinear Anal., 69 (9) (2008), 3153-3159.

[24] Kellermann, H. And Hieber, M., Integrated semigroup, J. Funct. Anal., 84 (1989), $160-180$.

[25] Kilbas, A.A., Srivastava, H.M. And Trujillo, J.J., Theory and Applications of Fractional Differential Equations, Elsevier Science B.V., Amsterdam, 2006.

[26] Kolmanovskit, V. AND Myshkis, A., Introduction to the Theory and Applications of Functional-Differential Equations. Kluwer Academic Publishers, Dordrecht, 1999.

[27] Lakshmikantham, V., Leela, S. and Vasundhara, J., Theory of Fractional Dynamic Systems, Cambridge Academic Publishers, Cambridge, 2009.

[28] Lakshmikantham, V. And Devi, J.V., Theory of fractional differential equations in a Banach space, Eur. J. Pure Appl. Math., 1 (2008), 38-45.

[29] Mainardi, F., Fractional calculus: Some basic problems in continuum and statistical mechanics, in: "Fractals and Fractional Calculus in Continuum Mechanics" (A. Carpinteri, F. Mainardi Eds.), Springer-Verlag, Wien, 1997, pp. 291-348.

[30] Metzler, F., Schick, W., Kilian, H.G. and Nonnenmacher, T.F., Relaxation in filled polymers: a fractional calculus approach, J. Chem. Phys., 103 (1995), 7180-7186.

[31] Miller, K.S. And Ross, B., An Introduction to the Fractional Calculus and Differential Equations, John Wiley, New York, 1993.

[32] MönCH, H., Boundary value problems for nonlinear ordinary differential equations of second order in Banach spaces, Nonlinear Anal., 4 (1980), 985-999.

[33] Mophou, G.M., Nakoulima, O. and N’Guérékata, G.M., Existence results for some fractional differential equations with nonlocal conditions, Nonlinear Studies, 17(1) (2010), 15-22. 
[34] Mophou, G.M. And N'GuéréKata, G.M., Existence of mild solution for some fractional differential equations with nonlocal conditions, Semigroup Forum, 79(2) (2009), $315-322$.

[35] Mophou, G.M. And N'GuéréKata, G.M., On integral solutions of some fractional differential equations with nondense domain, Nonlinear Analysis, T.M.A., 71(10) (2009), 4668-4675.

[36] Mophou, G.M. AND N'GuÉRÉKata, G.M., Mild solutions for semilinear fractional differential equations, Electron. J. Diff. Equ., Vol. 2009 (2009), No. 21, pp. 1-9.

[37] Mönch, H. And Von HaRten, G.F., On the Cauchy problem for ordinary differential equations in Banach spaces, Archiv. Math. Basel, 39 (1982), 153-160.

[38] PAZY, A., Semigroups of Linear Operators and Applications to Partial Differential Equations, Springer-Verlag, New York, 1983.

[39] Podlubny, I., Fractional Differential Equations, Academic Press, San Diego, 1999.

[40] Samko, S.G., Kilbas, A.A. And Marichev, O.I., Fractional Integrals and Derivatives. Theory and Applications, Gordon and Breach, Yverdon, 1993.

[41] Szufla, S., On the application of measure of noncompactness to existence theorems, Rend. Sem. Mat. Univ. Padova, 75 (1986), 1-14.

[42] Wu, J., Theory and Applications of Partial Functional Differential Equations, SpringerVerlag, New York, 1996. 